\title{
How to Communicate Uncertain Information Towards Clients as a Professional Service Provider: The Role of Uncertainty Disclosure and Communication Style: An Abstract
}

\section{Daniel Maar and Dirk Totzek}

\begin{abstract}
Professional service providers regularly have to make advice without knowing which course of action will finally work out and best address their clients' needs. It is unclear how professional service providers can effectively communicate suchlike uncertainties towards clients. To address this issue, we conducted a multimethod study. We first conducted semi-structured interviews with 30 representatives from different professional service industries. We also conducted two experiments in the consulting and health care context. Based on the interviews, we conceptualize typical strategies of uncertainty disclosure (open vs. restrictive) and communication styles (Dictator vs. Mentor vs. Motivator vs. Friend) of professional service providers. We then empirically analyze how these factors affect key client-related outcomes (i.e., service encounter satisfaction, satisfaction, trust, loyalty) before and after a service failure. We also consider the mediating role of clients' performance expectations. Our results indicate that professional service providers should employ the Friend communication style that aims at building a personal relationship with clients and at integrating them in the process of service provision. We show that communicating as Friend leads to more or equally favorable client outcomes than the other communication styles. Moreover, restrictive uncertainty disclosure tends to outperform open uncertainty disclosure. Particularly in the consulting context, Friends' client outcomes are not contingent on how uncertainty is disclosed. In addition, restrictive uncertainty disclosure can amplify the negative effect of a service failure in the health care arena. This might be due to the nature of such failures, which often directly and personally affect the client's medical condition.
\end{abstract}

References Available Upon Request

D. Maar $(\bowtie) \cdot$ D. Totzek

University of Passau, Passau, Germany

e-mail: daniel.maar@uni-passau.de; dirk.totzek@uni-passau.de

(C) Academy of Marketing Science 2017 